\title{
Novel Elongator Protein 2 Inhibitors Mitigating Tumor Necrosis Factor- $\alpha$ Induced Osteogenic Differentiation Inhibition
}

\author{
Wen-Jiao Wu, ${ }^{1}$ Chang-Liang Xia, ${ }^{2}$ Shuan-Ji Ou, ${ }^{2}$ Yang Yang, ${ }^{2}$ Yun-Fei Ma, ${ }^{3}$ Yi-Long Hou, ${ }^{3}$ \\ Qing-Po Yang, ${ }^{4}$ Jun Zhang, ${ }^{4}$ Jian-Wei Li, ${ }^{5}$ Yong Qi $\odot,{ }^{2}$ and Chang-Peng Xu $\oplus^{2}$ \\ ${ }^{1}$ Department of Medical Research Center, Guangdong Second Provincial General Hospital, Guangzhou, Guangdong, China \\ ${ }^{2}$ Department of Orthopaedics, Guangdong Second Provincial General Hospital, Guangzhou, Guangdong, China \\ ${ }^{3}$ Department of Orthopaedics and Traumatology, Nanfang Hospital, Southern Medical University, Guangzhou, Guangdong, China \\ ${ }^{4}$ Department of Orthopaedics, The First People's Hospital of Kashgar Prefecture, Kashgar, Xinjiang, China \\ ${ }^{5}$ Department of Orthopaedics, Shenzhen Shekou People's Hospital, Shenzhen, Guangdong, China
}

Correspondence should be addressed to Yong Qi; yongqi1979@163.com and Chang-Peng Xu; 731643426@qq.com

Received 20 August 2021; Accepted 12 October 2021; Published 22 November 2021

Academic Editor: Fabiano Bini

Copyright (C) 2021 Wen-Jiao Wu et al. This is an open access article distributed under the Creative Commons Attribution License, which permits unrestricted use, distribution, and reproduction in any medium, provided the original work is properly cited.

\begin{abstract}
Tumor necrosis factor- $\alpha$ is a common cytokine that increases in inflammatory processes, slows the differentiation of bone formation, and induces osteodystrophy in the long-term inflammatory microenvironment. Our previous study confirmed that the Elongation protein 2 (ELP2) plays a significant role in osteogenesis and osteogenic differentiation, which is considered a drug discovery target in diseases related to bone formation and differentiation. In this study, we applied an in silico virtual screening method to select molecules that bind to the ELP2 protein from a chemical drug molecule library and obtained 95 candidates. Then, we included 11 candidates by observing the docking patterns and the noncovalent bonds. The binding affinity of the ELP2 protein with the candidate compounds was examined by SPR analysis, and 5 out of 11 compounds performed good binding affinity to the mouse ELP2 protein. After in vitro cell differentiation assay, candidates 2\# and 5\# were shown to reduce differentiation inhibition after tumor necrosis factor- $\alpha$ stimulation, allowing further optimization and development for potential clinical treatment of inflammation-mediated orthopedic diseases.
\end{abstract}

\section{Introduction}

Persistent bone tissue inflammation, such as bone fractures and rheumatoid arthritis (RA), destroys the bone formation and absorption balance, which reduces bone mass and results in significant impact on bone regeneration [1]. So far, no effective drugs can cope with this clinical problem. We have noticed that elevated tumor necrosis factor-a (TNF- $\alpha$ ) is a major contributor to the bone pathophysiology through inhibition of osteoblasts function and stimulation of bone resorption activity of osteoclasts $[2,3]$. Drug for lessening the potential damage caused by longterm TNF- $\alpha$-associated inflammation exposure is urgent for clinical orthopedics.

Elongation protein 2 (ELP2) is a subordinate of the ELP123 complex and regulates the activity of elongation protein by integrating signals from various factors. It affects the inflammatory response through the JAK-STAT3 cascade and downstream pathways [4]. Our previous research identified the role of ELP2 in regulating TNF- $\alpha$-induced osteoblast differentiation inhibition. A mechanistic study concluded that ELP2 blocked the osteogenic differentiation induced by BMP-2 through activation the STAT3 pathway and downregulating the utterance of BMPR2, thereby slowing the process of early bone tissue formation in the inflammatory microenvironment [5]. Thus, ELP2 was shown to be the potential drug target to develop novel therapeutic for inflammation-induced bone loss.

In silico screening methods, such as quantitative structure-activity relationships (QSAR), pharmacophore models, molecular dynamics (MD) simulations, and highthroughput molecular docking, have been used to find 
specific protein inhibitors in recent research [6-9]. In our study, we used the molecular docking method to screening novel structure-specific chemicals for ELP2 and obtained eleven chemical compounds, which showed effective binding affinity to ELP2 active pockets. Combined with label-free surface plasmon resonance (SPR) affinity analysis, five candidates showed obvious binding affinity to ELP2. After in vitro experiments, two of the candidate compounds (2 \# and $5 \#$ ) were shown to block the retardation of osteoblast differentiation induced by TNF- $\alpha$, leading a potent clinical treatment potential for inflammation-induced bone loss.

\section{Materials and Method}

2.1. Protein and Ligand Library Preparation. The target protein was obtained from the UniProtKB database (https:// www.uniprot.org/) with an entry ID of Q91WG4 (Mus musculus), and the homocrystal structure was selected with the advanced sequence searching function aiming for high similarity. Then, the raw composition of the ELP2 was got from the database (https://www.rcsb.org/) with the identification number $5 \mathrm{M} 2 \mathrm{~N}$. The homobuilding step was performed with Structure Prediction Wizard in the Schrodinger software (Maestro, version 2015, Schrödinger LLC, USA). The missing loops and mismatches were corrected using a knowledge-based algorithm provided with the wizard module. To ensure the correct starting structure, the initial structure of the protein 3D model was prepared using the Protein Preparation Wizard module. Hydrogen atoms were increase in structure consistently with physiological $\mathrm{pH}$ (7.0). Then, the structure was optimized by adjusting hydrogen bonding and removing atom collisions, and formal charges are added to the heteron and optimized at neutral pH. Finally, the redundant water molecules were removed, and the new structure was minimized using the OPLS-2005 force field optimization potential. The prepared protein structure was subjected to binding site calculation.

The binding sites were generated using the SiteMap tool of the Schrödinger comprehensive assessed according to the calculated attributes, including the size of the site, the degree of protein enclosure, the degree of solvent exposure, the degree of tightness of site interaction with the protein, and the hydrophilic and hydrophobic character of the site. The total site scores were then used as the main basis to select potential binding sites. Finally, we completed the receptor grid generation step ranked the sitemaps from high to low score.

The chemical ligand library, including 552,007 compounds, was retrieved from the InterBioScreen database (InterBioScreen ltd., Russia) in structure data file (.SDF) format (Mar 2018 version). The molecules were arranged to ligand preparation using the LigPrep module with default settings. The ligand preparation process involved saving the definite chiralities and generating five minimum lowenergy stereoisomers per ligand with $\mathrm{pH}$ values ranging from 5.0 to 9.0 .

2.2. Virtual Screening. During the docking stage, the ligands were docked to the grids by three-tire-docking [10], which started with HTVS, followed by SP and XP to improve the accuracy. Then, we screened and chose the ligand according to the ranking of the GLIDE score. Herein, the optimized formula of the GLIDE score was as follows: GLIDE score $(\mathrm{kcal} / \mathrm{mol})=0.065 \times \mathrm{vdW}+0.130 \times \mathrm{Coul}+\mathrm{Lipo}+\mathrm{Hbond}+$ metal + BuryP + RotB + site [11]. The ADME properties of the selected ligands were analyzed using the QikProp tool to conclude the natural properties of the drugs, such as hydrogen bond donor and acceptor even the number of rotatable bonds.

2.3. Surface Plasmon Resonance (SPR) Assay. To validate the binding affinity of the candidate compounds with ELP2 protein, we tested the binding affinity through SPR assay. The experiment was performed as previous reported [12]. Briefly, the candidate molecules are crossed onto the chip surface by hydrogen substitution reaction. During the experimental SPR, the chips were first primed with running buffer ( $1 \mathrm{x}$ PBS containing 5\% DMSO). Mouse ELP2 protein used in the assay was diluted with running buffer at the concentrations from $200 \mathrm{nM}$ to $3200 \mathrm{nM}$. To validate the reliability of our method, we selected biotin and rapamycin binding with FKBP12 as a system control. Additionally, the solvents DMSO for compounds and PBS $(\mathbf{p H}=7.0)$ for proteins were measured individually as blank controls and background noise controls.

2.4. Cell Culture and Treatment. C2C12 cells were kept in DMEM complemented with the $10 \%$ fetal dairy serum and $1 \%$ penicillin/streptomycin and MC3T3-E1 cells in minimally needed culture medium (MEM, HyClone, GE Healthcare, USA) supplemented with $10 \%$ fetal bovine serum and $1 \%$ penicillin/streptomycin, were grown individually, and then cultured at $37.0^{\circ} \mathrm{C}, 5 \% \mathrm{CO}_{2}$. As previously reported [12], cells were cultured for differentiation induction in the presence of TNF- $\alpha$ with or without our candidate compounds.

2.5. Alkaline Phosphatase Measurement. Bone marrow transplantation was detected by ALP activity via the alkaline phosphatase assay kit followed by the instruction. A BioTek microplate spectrophotometer was used to assay the apparent density at the wavelength of $405 \mathrm{~nm}$. ALP activity values are equally common in the density protein found in stem cells determined to use the BCA Protein Assay Kit.

2.6. Mineralization Assessment. As reported, in two differentiation cell models, the culture medium was replaced every 3 days till 35th day. Mineralization of the cell was found by staining using the Optimized Alizarin Red S Stain Kit by the kit instructions.

2.7. Real-Time Fluorescent Quantitative PCR. The total mRNA was extracted from cell culture using TRIzol reagent (Thermo Scientific USA). The concentration and quality of the RNA were checked using a NanoDrop ${ }^{\mathrm{TM}} 8000$ spectrophotometer, and cDNA was then synthesized. Gene expression according to the recommendations of the reverse transcription kit was detected. Host gene $\beta$-actin was selected as the reference gene, and the gene expression level analysis was calculated by the $2^{-\Delta \Delta \mathrm{Ct}}$ quantification method [13]. 


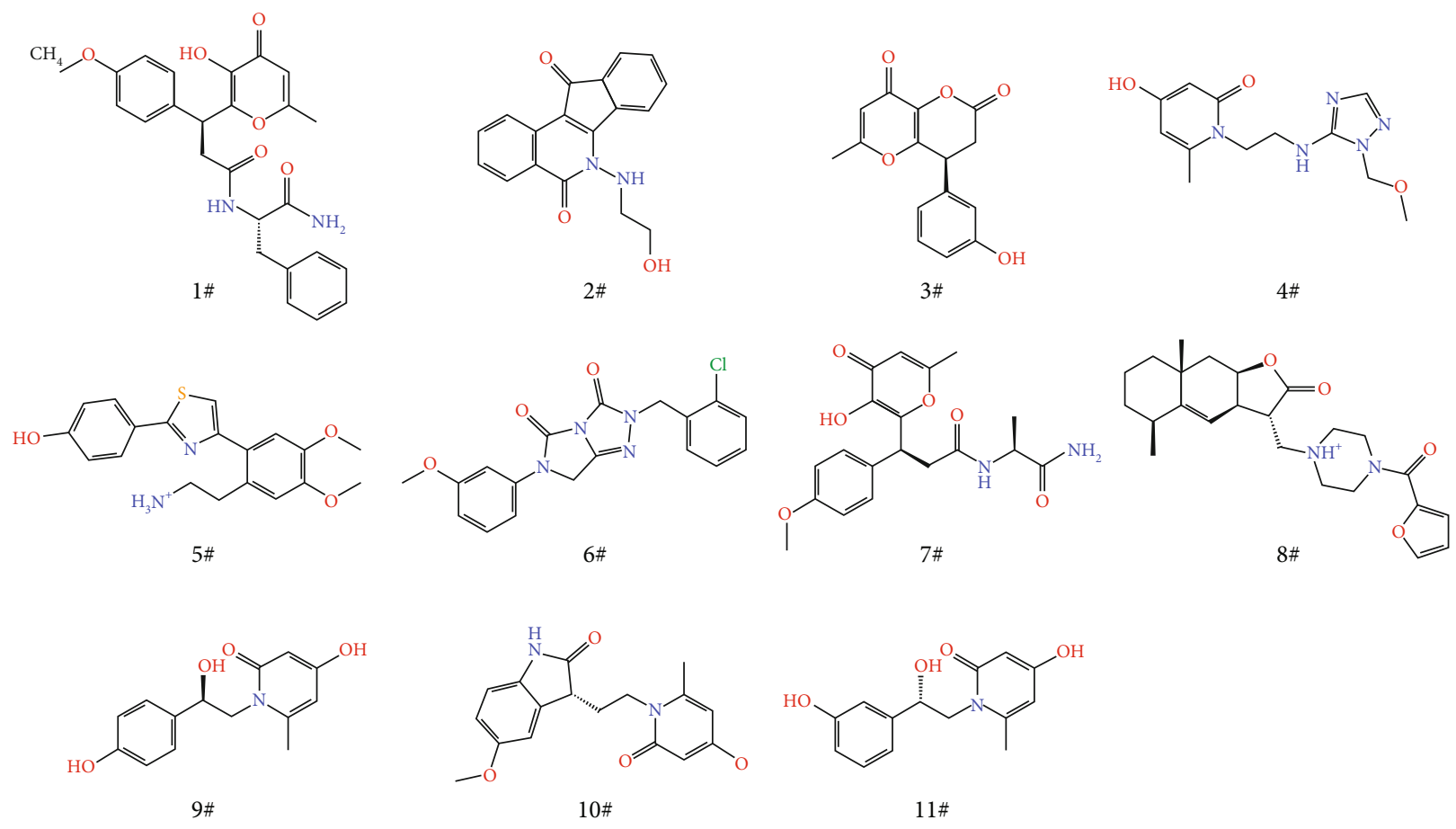

Figure 1: The chemical structures of 11 candidate compounds.

2.8. Western Blot Assay. After treatment, using PBS to wash the cells, the cells were lysed with RIPA lysis buffer. The cell lysate was then separated by SDS-PAGE. All proteins were transferred to PVDF membranes (Bio-Rad, USA). The PVDF membrane was then blocked with 5\% skim milk in Tris-buffered physiological saline $-0.1 \%$ Tween-20 for 1 hour and incubated with the following primary antibody: anti-ALP antibody (rabbit), anti-COL-1 antibody (mouse), anti-OCN antibody (mouse), anti-BMP-2 antibody (mouse), anti-Runx2 antibody (mouse), Anti-Osterix (rabbit), and anti- $\beta$-actin (mouse), followed by suitable horseradish peroxidase- (HRP-) labeled secondary antibody, blocking buffer for goat anti-rabbit IgG. Images were captured using G\&E imaging system.

2.9. Statistical Analysis. Multigroup comparison has been made with the two ANOVA methods. A single comparison was made using a one-sided Student's test $(P<0.05$ was counted as significance). Analysis was completed using SPSS 13.0 Statistics Package and Background 2017. The data is displayed as mean $\pm \mathrm{SD}$. The test was tripled and at least once.

\section{Results}

3.1. Modelling Preparation and Virtual Screening. The mouse ELP2 protein structure was homobuilt using PDB entry $5 \mathrm{M} 2 \mathrm{~N}$. Missing loops were filled and mismatches were corrected through a knowledge-based algorithm. A preliminary model was then set up using the Protein Preparation Wizard. After further structure optimization, binding sites were generated and receptor grids were calculated by a grid generation module. The chemical ligand library consisting of 552,007 compounds was prepared with the LigPrep module, yielding five minimum low-energy stereoisomers per ligand with $\mathrm{pH}$ values ranging from 5.0 to 9.0. In the ligand-docking stage, the ELP2 model and the entire ligand library were first applied to perform a high-throughput virtual screening (HT-VS), and 11,216 compounds were passed. These screened compounds were further analyzed by the standard precision (SP) and extraprecision (XP) glide docking models. 397 hits belonging to 102 clusters were retrieved from the Glide SP docking with a docking score cut-off value of $-6.0 \mathrm{kcal} / \mathrm{mol}$. Subsequently, the Glide XP module was used to further reduce the hit set, and 95 hits belonging to 36 clusters were retrieved with a docking score cut-off value of $-5.0 \mathrm{kcal} / \mathrm{mol}$. Finally, these 95 ligand molecules were visually inspected based on docking poses and bonds interacting with protein residues. Finally, 11 hits were selected as candidate compounds. The structures of the 11 compound candidates are shown in Figure 1. The binding sites and docking surface structures are shown in Supplementary Figure $1 \mathrm{~A}$ and $1 \mathrm{~B}$.

3.2. SPR Affinity Analysis. Under the technique of photocross-linker biosensor chip, the candidate compounds were immobilized onto the chemically modified chip surface in random orientations and without any attached label or linker. After the compounds were fixed onto the surface of the chip, ELP2 protein solved in PBS were passed over the chip surface. The original sensor gram information was collected in real time. The association rate constants $\left(K_{\text {on }}\right)$, dissociation rate constants $\left(K_{\text {off }}\right)$, and the equilibrium dissociation constant (KD) of samples are shown in Table 1. The binding curves are shown in Figure 2. According to the affinity measurement results, five among the eleven candidates 
TABle 1: Affinity constants of candidate compounds with ELP2 protein.

\begin{tabular}{lccccc}
\hline No. & Protein & Compound & $\operatorname{Avg} K_{\text {on }}\left(\mathrm{M}^{-1} \mathrm{~s}^{-1}\right)$ & $\operatorname{Avg} K_{\text {off }}\left(\mathrm{s}^{-1}\right)$ & $\operatorname{Avg} \mathrm{KD}(\mathrm{M})$ \\
\hline $1 \#$ & Elp2 & $1 \#$ & $1.98 E+03$ & $2.34 E-01$ & $1.18 E-04$ \\
$2 \#$ & Elp2 & $2 \#$ & $1.65 E+02$ & $2.41 E-04$ & $1.46 E-06$ \\
$3 \#$ & Elp2 & $3 \#$ & $2.31 E+00$ & $4.50 E-01$ & $1.95 E-01$ \\
$4 \#$ & Elp2 & $4 \#$ & $1.85 E+00$ & $3.42 E-01$ & $1.85 E-01$ \\
$5 \#$ & Elp2 & $5 \#$ & $3.72 E+03$ & $1.22 E-02$ & $3.28 E-06$ \\
$6 \#$ & Elp2 & $6 \#$ & $2.54 E+00$ & $4.15 E-01$ & $1.63 E-01$ \\
$7 \#$ & Elp2 & $7 \#$ & $3.47 E+02$ & $1.10 E-03$ & $3.17 E-06$ \\
$8 \#$ & Elp2 & $8 \#$ & $1.12 E+04$ & $1.05 E-03$ & $9.36 E-08$ \\
$9 \#$ & Elp2 & $9 \#$ & $5.02 E+02$ & $7.50 E-02$ & $1.49 E-04$ \\
$10 \#$ & Elp2 & $10 \#$ & $2.18 E+00$ & $5.04 E-01$ & $2.32 E-01$ \\
$11 \#$ & Elp2 & $11 \#$ & $2.22 E+02$ & $7.74 E-04$ & $3.48 E-06$ \\
Blank & Elp2 & DMSO & $1.39 E+00$ & $9.03 E-01$ & $6.51 E-01$ \\
\hline
\end{tabular}

possessed strong binding affinity to ELP2, and another two candidates ( $1 \#$ and 9\#) showed much faster dissociation rates and were excluded due to presumable difficulty in maintaining adequate efficacy in vivo.

3.3. 2\# and 5\# Compounds Show Remarkable Mitigation against TNF- $\alpha$-Induced ALP Activity Inhibition. To determine the effect of the candidate compounds on TNF- $\alpha$ induced osteogenic differentiation inhibition, we examined the activity of osteogenic marker alkaline phosphatase in the cells. As mentioned in Materials and Method, cells were grown in an adequate osteoblast differentiation environment and tested the alkaline phosphatase activity. Staining observations and relative ALP activity are showed in Figures 3. The results indicated that compared with the osteogenic differentiation controls of the ID and SD differentiation models, TNF- $\alpha$ significantly reduced ALP activity and lightened the color after ALP staining, while the presence of candidates $2 \#$ and $5 \#$ brought a significant ease to TNF- $\alpha$ induced inhibition of osteoblast differentiation in $\mathrm{C} 2 \mathrm{C} 12$ (Figures 3(a) and 3(b)) and MC3T3-E1 cell (Figures 3(c) and $3(\mathrm{~d})$ ). The effects of candidates $7 \#, 8 \#$, and $11 \#$ were not as strong as $2 \#$ and $5 \#$, with only slightly easing of the inhibition resulting from TNF- $\alpha$ (data not shown). The half-effective concentration $\left(\mathrm{EC}_{50}\right)$ of candidates $2 \#$ and $5 \#$ were measured through examining the ALP activity (Figure 4); the $\mathrm{EC}_{50}$ of $2 \#$ candidate was $14.71 \mu \mathrm{M}$ in $\mathrm{C} 2 \mathrm{C} 12$ cells of the ID model; at the same time, the $\mathrm{EC}_{50}$ in the MC3T3-E1 SD model was $29.68 \mu M$. The $\mathrm{EC}_{50}$ values of $5 \#$ candidate were $12.15 \mu \mathrm{M}$ and $18.24 \mu \mathrm{M}$ in ID and SD model cells, respectively. Candidate 5\# possessed better protective effect compared with candidate $2 \#$, which was consistent with SPR binding affinity test result that 5\# showed higher binding affinity to ELP2 protein (Figure 2).

3.4. 2\# and 5\# Compounds Reverse TNF- $\alpha$-Induced Inhibition on Osteoblast Mineralization Activity. In order to test the function of the candidate compounds on the mineralization activity during osteoblasts differentiation,

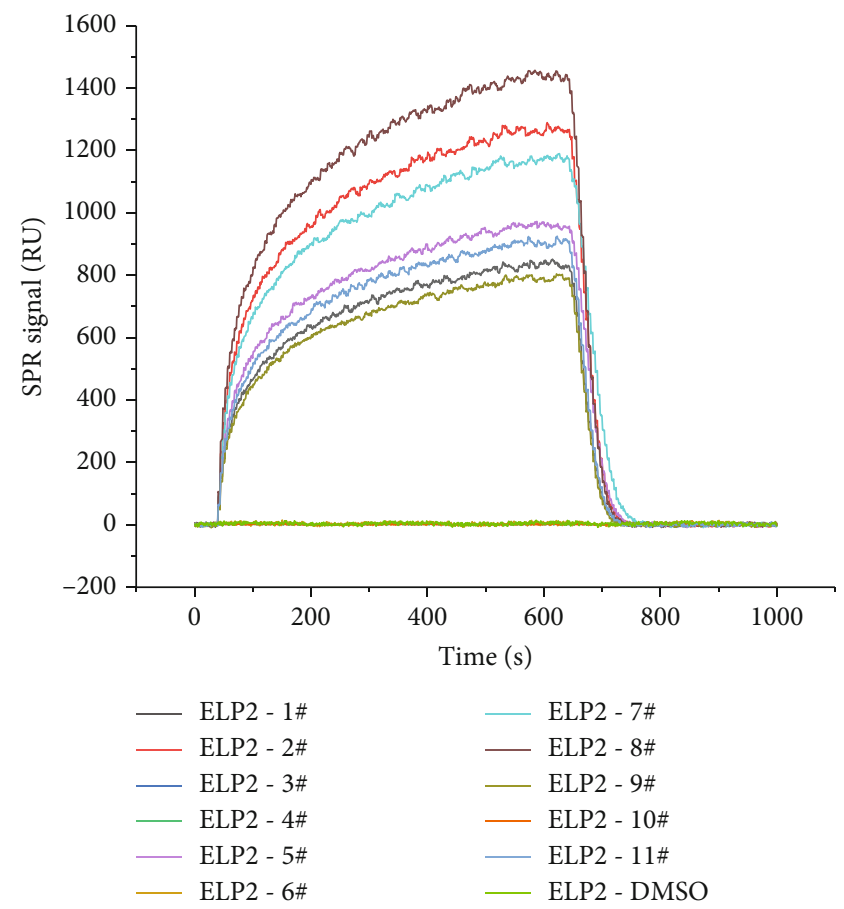

FIgURE 2: The binding curve of compound 1\# to 11 to the mouse ELP2 protein. The selected compounds had been fixed onto the chip surface; ELP2 protein solved in PBS were passed over the chip surface. The raw sensor gram information was collected in real time. The binding of each compound to the ELP2 protein during each cycle is represented by the response unit (RU) of surface resonance.

the self-differentiation (SD) model and inflammatorydifferentiated (ID) model cells were stained with Alizarin Red S staining on day 35 after differentiation induction, and the mineralization activity of each group can be estimated by the number of stained spots. As Figure 5 has shown, extracellular mineralization was stained with Alizarin Red S staining; compared with the TNF- $\alpha$ control 


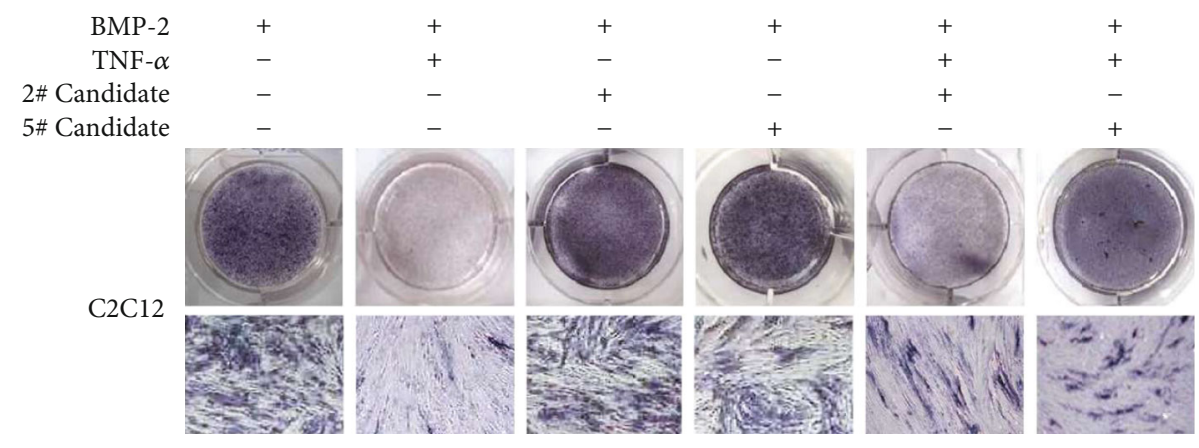

(a)

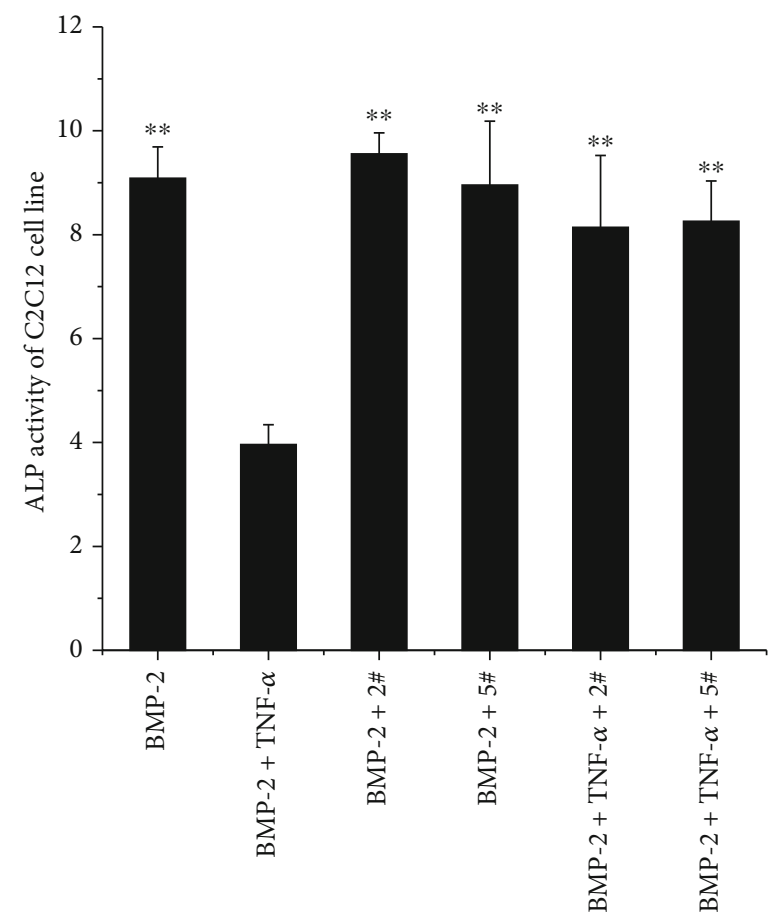

(b)

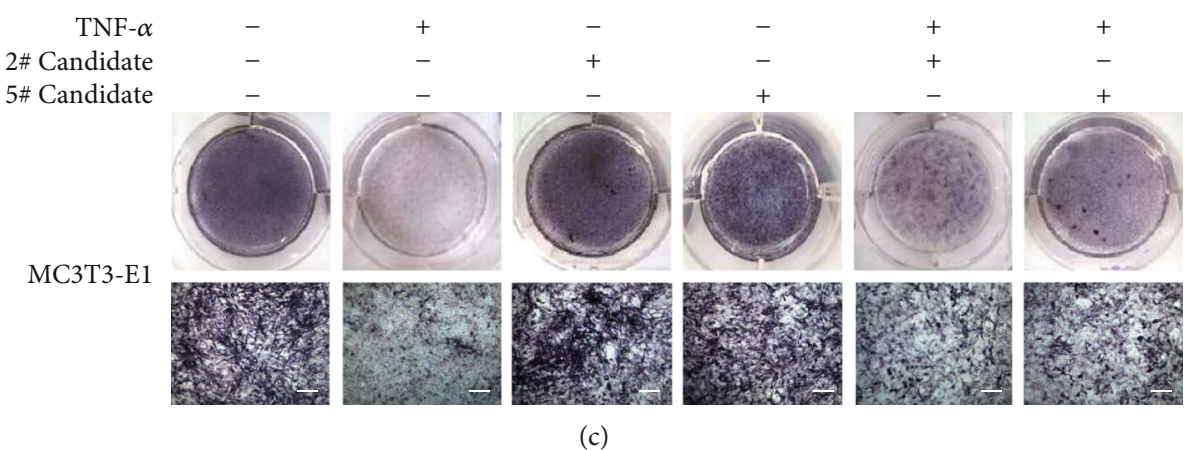

Figure 3: Continued. 


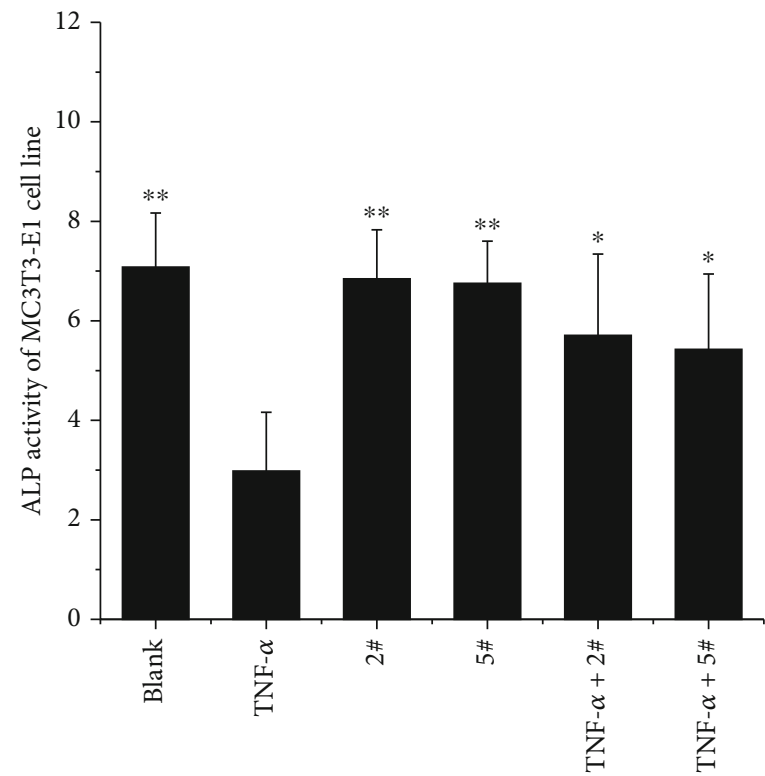

(d)

Figure 3: The ALP stain and activity measurements of 2\# and 5\# candidate on C2C12 and MC3T3-E1 cell osteogenic differentiations. 2\# and 5\# compounds show remarkable mitigation against TNF- $\alpha$ induced ALP activity inhibition. The ALP staining observation in each group $(\mathrm{a}, \mathrm{c})$. The relative ALP activity were measured from the cell lysates of each group (b, d). Data are mean $\pm \mathrm{SD} ;{ }^{*} P<0.05,{ }^{* *} P<0.01$, each experiment group vs. the TNF- $\alpha$ treatment cell group.

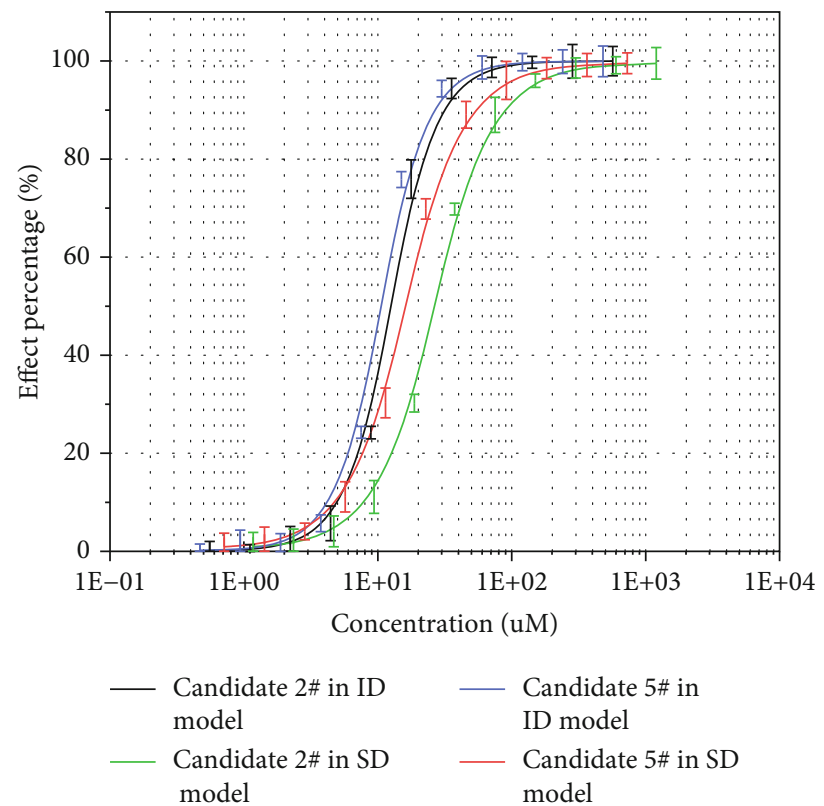

FIgure 4: The $\mathrm{EC}_{50}$ values of candidates $2 \#$ and 5\# were calculated and drafted as the dose-effect curves.

groups, candidates $2 \#$ and $5 \#$ increased the red spot on the cell surface, suggesting that cell mineralization can be reversed by the presence of candidates $2 \#$ and $5 \#$ in $\mathrm{C} 2 \mathrm{C} 12$ and MC3T3-E1 cells (Figure 5), and we inferred that the TNF- $\alpha$-induced osteoblast differentiation inhibition was reversed based on the appearance of mineralization activity changes in the cell models.
3.5. 2\# and 5\# Compounds Increased the Expression Level of Osteoblast Differentiation-Related Marker after TNF- $\alpha$ Stimulation. In order to investigate the changes in osteogenic differentiation associated molecules including COL-I, ALP, OCN, BMP-2, RUNX-2, and OSX genes, we performed Q-PCR and Western blot analysis on the differentiation cellular models on the 7th day after osteoblast cell 


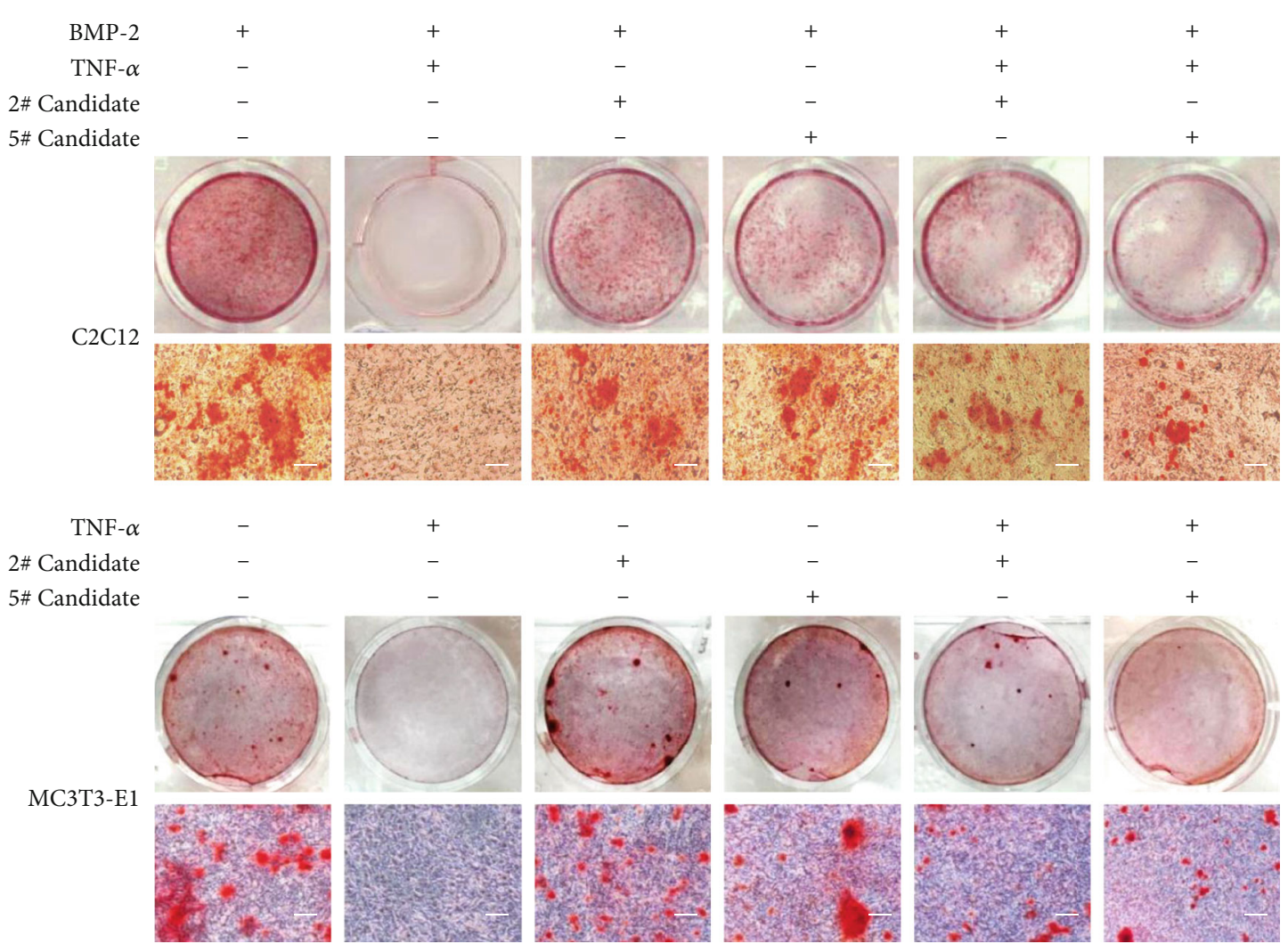

FIGURE 5: Alizarin Red S staining of differentiated osteogenic cells, the self-differentiation (SD) model, and inflammatory-differentiated (ID) model cells were stained with Alizarin Red S staining on day 35 after differentiation induction, and the mineralization activity of each group can be estimated by the number of stained spots $($ bar $=50 \mu \mathrm{m})$.

TAble 2: Primer sequences of osteogenic differentiation marker genes.

\begin{tabular}{|c|c|c|c|}
\hline Gene symbol & Primer sequence $\left(5^{\prime}-3^{\prime}\right)$ & Product size (bp) & Accession no. \\
\hline \multirow{2}{*}{ COL-I } & F: $5^{\prime}$-GACCCTAACCAAGGATGCAA-3' & \multirow{2}{*}{200} & \multirow{2}{*}{ NG_007404.1 } \\
\hline & R: 5'-GGAAGTTCAGGATTGCCGTA-3' & & \\
\hline \multirow{2}{*}{ ALP } & F: 5'-CCACGTCTTCACATTTGGTG-3' & \multirow{2}{*}{196} & \multirow{2}{*}{ NG_008940.1 } \\
\hline & R: 5' -AGACTGCGCCTGGTAGTTGT-3' & & \\
\hline \multirow{2}{*}{ OCN } & F: $5^{\prime}$-GTGCAGAGTCCAGCAAAGGT-3' & \multirow[b]{2}{*}{202} & \multirow{2}{*}{ NG_047015.1 } \\
\hline & R: 5'-CGATAGGCCTCCTGAAAGC-3' & & \\
\hline \multirow[b]{2}{*}{ BMP-2 } & F: $5^{\prime}$-TCAAGCCAAACACAAACAGC-3' & \multirow[b]{2}{*}{197} & \multirow{2}{*}{ NG_023233.1 } \\
\hline & R: 5' -ACGTCTGAACAATGGCATGA-3' & & \\
\hline \multirow{2}{*}{ RUNX-2 } & F: 5' -CTCTTCCCAAAGCCAGAGTG-3' & \multirow{2}{*}{206} & \multirow{2}{*}{ NG_008020.1 } \\
\hline & R: 5' -CAGCGTCAACACCATCATTC-3' & & \\
\hline \multirow{2}{*}{ OSX } & F: $5^{\prime}$-TAATGGGCTCCTTTCACCTG-3' & \multirow{2}{*}{198} & \multirow{2}{*}{ NG_023391.2 } \\
\hline & R: 5'-GAGCCATAGGGGTGTGTCAT-3' & & \\
\hline
\end{tabular}

differentiation are shown in Table 2. According to the difference of the gene expression levels, the differentiation control group was significantly upregulated compared with TNF- $\alpha$ treated cells on the day 7 . The level of above gene expression was raised in the $2 \#$ and the $5 \#$ candidate-treated cell with cell morphology phenotype differentiation when compared to the cell stimulated with TNF- $\alpha$ (Figure 6). A further study of the expression of osteoblastic markers at the protein level was performed using Western blot. As shown in Figure 7, the expressions of the marker proteins were consistent with 


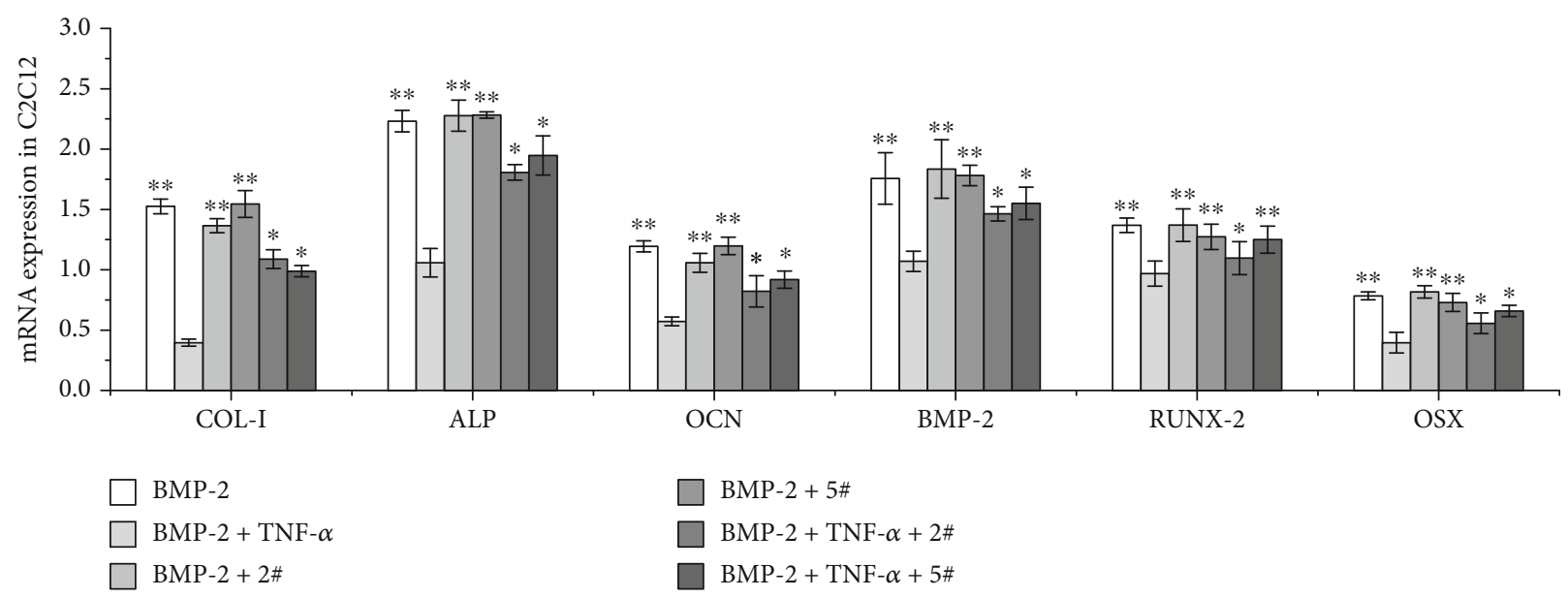

(a)

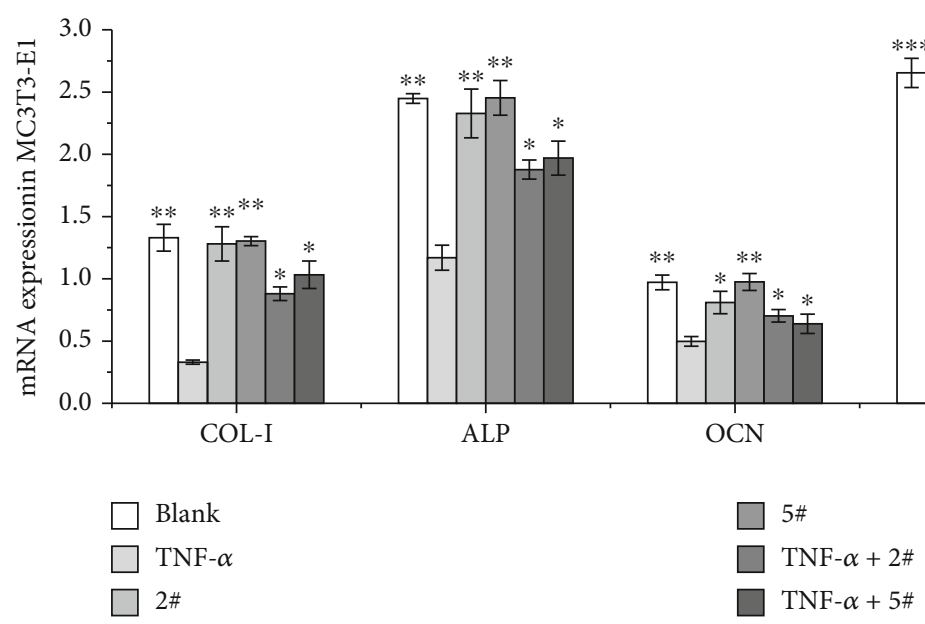

(b)

FIGURE 6: Effect of 2\# and 5\# treatment on TNF- $\alpha$-stimulated C2C12 (a) and MC3T3-E1 (b) cell osteoblast differentiation-associated marker gene expression. Q-PCR analysis in the differentiation was conducted on the 7th day after osteoblast cell differentiation in the ID and SD models. Data are mean $\pm \mathrm{SD} ;{ }^{*} P<0.05,{ }^{* *} P<0.01$, each experiment group vs. the TNF- $\alpha$ group.

the mRNA expressions. Therefore, our above experiments indicated that the 2\# and 5\# ELP2 inhibitor candidates could effectively lighten the TNF- $\alpha$-induced inhibition of primary osteoblast differentiation during bone inflammation.

\section{Discussion}

With different bone cells working together, it completes the role of absorbing the old bone and generating a new bone. On this way, the morphology and structure of the bone will be constantly destroyed and remodeled, and this depends on the number and proportion of cells, so the differentiation of osteoblasts can affect the morphology and structure of bone. Persistent inflammatory bone environment hinders osteoblastic differentiation, leading to bone dysplasia [14-16]. TNF- $\alpha$ is a main contributor to persistent inflammationand infection-induced bone regeneration inhibitions; the underline mechanism is still not clear [17]. A previous study [6] reported that ELP2 positively regulated the increased
TNF- $\alpha$-induced repression of osteoblast differentiation during persistent inflammation. In clinical studies, the repression of osteoblast differentiation will affect the regeneration and formation of bone. Therefore, ELP2 can inhibit bone dysplasia induced by TNF- $\alpha$, which leads it playing a role in long-term chronic infections.

Molecular docking methods are an effective method to identify potential chemical structures for novel targets [18]. Compared with traditional high-throughput screening, the computational calculation method is faster and more costeffective. The Schrödinger suit is also an efficient ligand screening program, which is widely used and has been proven in the field of ligand-seeking research $[19,20]$. In this study, we performed virtual docking following the published method and using the IBS compound library and obtained 11 candidates that fit into the active site of the homobuilt mouse ELP2 protein. These candidate compounds were prepared for SPR affinity analysis with ELP2 protein, which is a technique that enables rapid detection of the interaction 


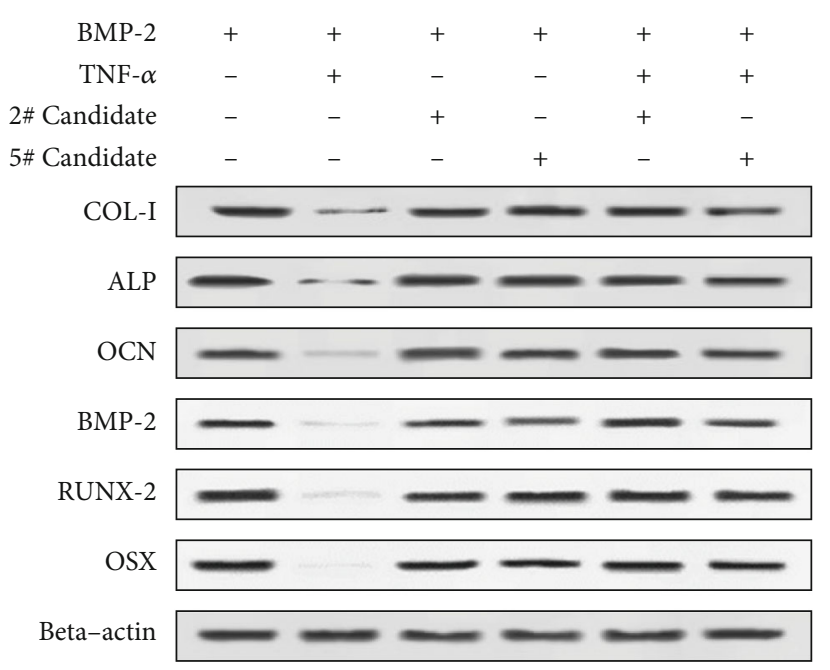

(a)

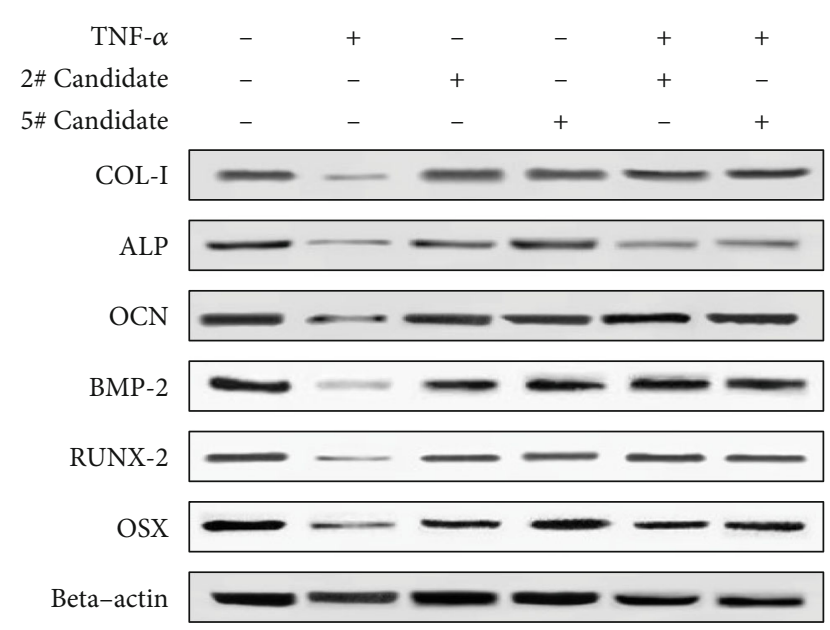

(b)

Figure 7: The expression of osteogenic differentiation markers were increased by the 2\# and 5\# drug candidates compared with TNF- $\alpha$ treatment in the cell differentiation process. The protein of the differentiation model cells was harvested on the 7 th day after osteoblast cell differentiation and then subjected to Western blot analysis.

between two molecules together with their binding kinetic parameters in real time [21]. Up to now, no research has reported the epitope of ELP2 active center; therefore, we simulated some possible active centers using the simulation method with the protein structure building module. It is undeniable that such a method results in a large number of incorrect active centers and ineffective ligand candidates in the screening. We speculated that this was the reason only 2 of the 11 high-scored candidate compounds showed inhibition activity in the in vitro experiments.

During our experiment, we applied an in silico virtual screening to select compound molecules from the chemical drug library that bind to the ELP2 protein and obtained 95 candidates. We further filtered out 11 candidates by scoring the docking model and observing noncovalent bonds. Afterwards, the binding affinities of the ELP2 protein with candidate compounds were analyzed by SPR assay, and 5 of the 11 compounds possessed obvious binding affinity to ELP2 protein. Accordingly, these 5 potential compounds were used for the in vivo efficiency study to determine whether there was an associated potential drug effect. The candidate was tested in the differentiation model and verified, which are used for evaluation of osteoblast differentiation [22]. Further, Q-PCR and Western blot analyses were used to investigate the osteogenic differentiation markers which was affected by the two compounds; the results were consistent with previous, which indicated that 2 compounds could mitigate the effect of TNF- $\alpha$-induced differentiation inhibition.

In summary, we obtained two competitive inhibitors of ELP2 protein; both of them have the potential to impede TNF- $\alpha$-induced new bone-forming osteoblasts (OB) inhibition in two cells. The $\mathrm{EC}_{50}$ of candidate 2 \# was $14.71 \mu \mathrm{M}$ in the $\mathrm{C} 2 \mathrm{C} 12$ cells of the ID model, while the $\mathrm{EC}_{50}$ in the MC3T3-E1 SD model was $29.68 \mu \mathrm{M}$. Candidate 5 \# EC 50 values were $12.15 \mu \mathrm{M}$ and $18.24 \mu \mathrm{M}$ in the ID and SD model cells, respectively. The results about the bone differentiation assay were in line with expectations. The results give hope that the 2 \# and 5 \# candidates will become new drugs in clinical orthopedics. Finally, further research will spare no effort to clarify the additional pharmacodynamics and metabolic kinetics of the 2\# and 5\# candidates, so as to develop them into clinical drugs that prevent the inhibition of bone regeneration caused by persistent inflammation.

\section{Abbreviations}

COL-I: Collagen alpha-1

$\mathrm{EC}_{50}$ : $\quad$ Effective concentration 50

EC: $\quad$ Elongator complex

ELP123: Elongator subunits 1-3

ELP456: Elongator subunits 4-6

ELP2: $\quad$ Elongator protein 2

FBS: $\quad$ Fetal bovine serum

GLIDE: Grid-based ligand docking with energetics

HRP: $\quad$ Horseradish peroxidase

HTVS: High-throughput virtual screening

ID model: Inflammatory differentiation model

KD: $\quad$ Dissociation constant, $K_{\text {on }} / K_{\text {off }}$

MD: $\quad$ Molecular dynamics

OCN: Osteocalcin

OSX: Osterix

Pol II: $\quad$ RNA polymerase II

QSAR: Quantitative structure-activity relationships

RA: Rheumatoid arthritis

RU: $\quad$ Response units

RUNX-2: Runt-related transcription factor 2

SD model: Self-differentiation model

SLE: $\quad$ Systemic lupus erythaematosus

SP: $\quad$ Standard precision

SPR: $\quad$ Surface plasmon resonance

StIP1: $\quad$ STAT3-interacting protein 1

XP: $\quad$ Extraprecision mode. 


\section{Data Availability}

Once upon request, the corresponding author will offer the data included in this study.

\section{Conflicts of Interest}

The authors declared no conflicts of interests to this study.

\section{Authors' Contributions}

Wen-Jiao Wu and Chang-Liang Xia contributed equally to this work. Wen-Jiao $\mathrm{Wu}$ and Chang-Liang Xia did the experiment and contributed equally to this research and should be regarded as co-first author. Chang-Peng $\mathrm{Xu}$ and Yong Qi contribute to the experimental plan design and funding application. The main work of Shuan-Ji Ou, Yang Yang, Yun-Fei Ma, and Yi-Long Hou is in silico docking and data analysis, including chart making and figure design. The work of Qing-Po Yang, Jun Zhang, and Jian-Wei Li was reporting of the work, including drafting, copywriting, and revising.

\section{Acknowledgments}

This work was supported by the National Natural Science Foundation of China (No. 81972083), Science and Technology Planning Project of Guangzhou (Nos. 202102080052, 202102010057, and 201804010226), Science Foundation of Guangdong Second Provincial General Hospital (Nos. 3DA2020004, 3D-A2020002, YQ2019-009, and 2019BSGZ012), Natural Science Foundation of Xinjiang Province (No. 2018D01C014), and Minority Science and Technology Talent Special Training Program Project of Xinjiang Province (No. 2019D03025).

\section{Supplementary Materials}

Supplementary Figure 1: the binding sites and docking surface structures of eleven candidates in docking procedure. (Supplementary Materials)

\section{References}

[1] S. H. McBride-Gagyi, J. A. McKenzie, E. G. Buettmann, M. J. Gardner, and M. J. Silva, "Bmp2 conditional knockout in osteoblasts and endothelial cells does not impair bone formation after injury or mechanical loading in adult mice," Bone, vol. 81, pp. 533-543, 2015.

[2] M. Almeida, M. R. Laurent, V. Dubois et al., "Estrogens and androgens in skeletal physiology and pathophysiology," Physiological Reviews, vol. 97, no. 1, pp. 135-187, 2017.

[3] M. S. Nanes, "Tumor necrosis factor- $\alpha$ : molecular and cellular mechanisms in skeletal pathology," Gene, vol. 321, pp. 1-15, 2003.

[4] M. I. Dauden, J. Kosinski, O. Kolaj-Robin et al., "Architecture of the yeast Elongator complex," EMBO Reports, vol. 18, no. 2, pp. 264-279, 2017.

[5] C. P. Xu, X. Li, Y. J. Hu et al., "Quantitative proteomics reveals ELP2 as a regulator to the inhibitory effect of TNF- $\alpha$ on oste- oblast differentiation," Journal of Proteomics, vol. 114, no. 69, pp. 234-246, 2015.

[6] P. D. Patel, M. R. Patel, N. Kaushik-Basu, and T. T. Talele, "3D QSAR and molecular docking studies of benzimidazole derivatives as hepatitis C virus NS5B polymerase inhibitors," Journal of Chemical Information and Modeling, vol. 48, no. 1, pp. 42-55, 2008.

[7] T. T. Talele, P. Arora, S. S. Kulkarni et al., "Structure-based virtual screening, synthesis and SAR of novel inhibitors of hepatitis C virus NS5B polymerase," Bioorganic \& Medicinal Chemistry, vol. 18, no. 13, pp. 4630-4638, 2010.

[8] Y. Deng, G. W. Shipps Jr., T. Wang et al., "Discovery of 4 _H_ -pyrazolo[1,5- _a_ ]pyrimidin-7-ones as potent inhibitors of hepatitis $C$ virus polymerase," Bioorganic \& Medicinal Chemistry Letters, vol. 19, no. 18, pp. 5363-5367, 2009.

[9] S. Yan, G. Larson, J. Z. Wu et al., "Novel thiazolones as HCV NS5B polymerase allosteric inhibitors: further designs, SAR, and X-ray complex structure," Bioorganic \& Medicinal Chemistry Letters, vol. 17, no. 1, pp. 63-67, 2007.

[10] S. Gudipati, R. M, A. Mankad, H. Pandya, and Y. T. Jasrai, "Molecular docking based screening of Noggin inhibitors," Bioinformation, vol. 14, no. 1, pp. 15-20, 2018.

[11] K. Dhanachandra Singh, P. Kirubakaran, S. Manikandaprabhu, S. Nagamani, P. Srinivasan, and M. Karthikeyan, "Docking studies of adenosine analogues with NS5 methyltransferase of yellow fever virus," Indian Journal of Microbiology, vol. 52, no. 1, pp. 28-34, 2012.

[12] C. Xu, Y. Qi, Z. Cui et al., "Discovery of novel elongator protein 2 inhibitors by compound library screening using surface plasmon resonance," RSC Advances, vol. 9, no. 3, pp. 16961704, 2019.

[13] J. S. Yuan, A. Reed, F. Chen, and C. N. Stewart Jr., "Statistical analysis of real-time PCR data," BMC Bioinformatics, vol. 7, no. 1, p. 85, 2006.

[14] C. Gao, S. Peng, P. Feng, and C. Shuai, "Bone biomaterials and interactions with stem cells," Bone Research, vol. 5, no. 1, p. 17059, 2017.

[15] A. Kassem, C. Lindholm, and U. H. Lerner, "Toll-like receptor 2 stimulation of osteoblasts mediates staphylococcus aureus induced bone resorption and osteoclastogenesis through enhanced RANKL," PLoS One, vol. 11, no. 6, article e0156708, 2016.

[16] D. V. Novack and G. Mbalaviele, "Osteoclasts-key players in skeletal health and disease," Microbiology spectrum, vol. 4, no. 3, 2016.

[17] E. Tsialogiannis, I. Polyzois, Q. O. Tang et al., “Targeting bone morphogenetic protein antagonists:in vitroandin vivoevidence of their role in bone metabolism," Expert Opinion on Therapeutic Targets, vol. 13, no. 1, pp. 123-137, 2009.

[18] D. R. Houston, L. H. Yen, S. Pettit, and M. D. Walkinshaw, "Structure- and ligand-based virtual screening identifies new scaffolds for inhibitors of the oncoprotein MDM2," PLoS One, vol. 10, no. 4, article e0121424, 2015.

[19] R. A. Friesner, J. L. Banks, R. B. Murphy et al., "Glide: a new approach for rapid, accurate docking and scoring. 1. Method and assessment of docking accuracy," Journal of Medicinal Chemistry, vol. 47, no. 7, pp. 1739-1749, 2004.

[20] R. A. Friesner, R. B. Murphy, M. P. Repasky et al., "Extra precision glide: docking and scoring incorporating a model of hydrophobic enclosure for protein-ligand complexes," Journal of Medicinal Chemistry, vol. 49, no. 21, pp. 6177-6196, 2006. 
[21] J. A. Salon, D. T. Lodowski, and K. Palczewski, "The significance of $\mathrm{G}$ protein-coupled receptor crystallography for drug discovery," Pharmacological Reviews, vol. 63, no. 4, pp. 901937, 2011.

[22] Z. Yu, P. Gauthier, Q. T. Tran et al., "Differential properties of human ALP+ periodontal ligament stem cells vs their ALPcounterparts," Journal of Stem Cell Research \& Therapy, vol. 5, 2015. 